\title{
Consommation alimentaire des ménages et déterminants de la diversité alimentaire : cas de quatre communes dans la région du Nord, Burkina Faso
}

\author{
Sita SANOU ${ }^{1 *}$, Augustine AYANTUNDE ${ }^{2}$ et Aimé Joseph NIANOGO ${ }^{3}$ \\ ${ }^{1}$ Institut de l'Environnement et de Recherches Agricoles (INERA), 01 BP 476, Ouagadougou, Burkina Faso/ \\ BP 1496 Ouagadougou, Burkina Faso. \\ ${ }^{2}$ International Livestock Research Institute (ILRI), BP 1496 Ouagadougou, Burkina Faso. \\ ${ }^{3}$ Université Nazi BONI (UNB), Institut de Développement Rural, 01 BP 1091, Fax 20972577 \\ *Auteur correspondant, E-mail : zouwiss@yahoo.fr, Tel: +22625333310.
}

\section{RESUME}

Une alimentation diversifiée est nécessaire pour apporter à l'organisme l'essentiel des nutriments dont il a besoin pour son fonctionnement. L'objectif de ce travail a été d'évaluer la consommation alimentaire des ménages dans la partie Nord du Burkina Faso et d'identifier les déterminants de la diversité alimentaire. L'évaluation de la diversité alimentaire a été réalisée à partir du calcul du score de diversité alimentaire des ménages à l'aide de l'outil RHoMIS (Rural Household Multiple Indicator Survey). Une enquête a été conduite auprès de 300 ménages pour caractériser le profil socio-économique et la consommation alimentaire au sein de ces ménages. La valeur moyenne du score de diversité alimentaire des ménages pour une période de référence de 7 jours, a été de 7,3 $(0,1)$ durant la période favorable et de $5,7(0,1)$ durant la période de soudure. Les céréales ont constitué l'aliment énergétique de base et le niébé a représenté l'essentiel des aliments protéiques, la consommation des produits d'origine animale étant faible. Les résultats montrent que le nombre de cultures produites par le ménage et les revenus agricoles ne sont pas positivement associés à la diversité alimentaire élevée. Seul le revenu provenant de l'orpaillage est associé positivement à une diversité alimentaire élevée.

(C) 2018 International Formulae Group. All rights reserved.

Mots clés : Diversité alimentaire, déterminants, sources de revenu, système agro-pastoral, Burkina Faso.

\section{Household food diversity and animal-source foods consumption in Burkina Faso: case of three communes in Yatenga at North of the country}

\begin{abstract}
A diversified diet is necessary to provide essential nutrients which the body needs for its functioning. The objective of this study was to evaluate the dietary consumption of households in the Northern part of Burkina Faso and to identify the determinants of dietary diversity. Household dietary diversity was assessed by calculating household dietary diversity score (HDDS). Three hundred households were surveyed in six communities in Northern Region of Burkina Faso to characterize the socio-economic profile and food consumption of the surveyed households. A HDDS was constructed according to the Rural Household Multiple Indicator Survey (RHoMIS). The HDDS was seven food groups during the normal period and about six food groups $(5.7 \pm 0.1)$ during the difficult food period. Cereals were the staple food and cowpea provided most of the dietary protein as the consumption of animal products was generally low. The results of the regression
\end{abstract}


model show that the number of food crops produced and farmers' farm cash receipts are not positively associated with high dietary diversity. Only income from gold mining contributed significantly to household dietary diversity.

(C) 2018 International Formulae Group. All rights reserved.

Keywords: Dietary diversity, food types, income sources, agro-pastoral system, Burkina Faso.

\section{INTRODUCTION}

Pour résoudre le problème d'insécurité alimentaire en Afrique sub-saharienne, l'accent est mis sur la production et la consommation des aliments énergétiques au détriment d'une diversité alimentaire (Jones et al., 2014). Il est clairement établi que la consommation de quantités suffisantes d'énergie n'est pas synonyme d'apports de nutriments nécessaires au bon fonctionnement de l'organisme (Rashid et al., 2011). De ce fait, en plus de fournir aux populations, une quantité suffisante de calories, il est nécessaire de leur permettre de disposer d'une diversité alimentaire garantissant les apports de nutriments nécessaires à une alimentation saine et équilibrée (Remans et al., 2014). La diversité alimentaire qui est une mesure de l'adéquation de l'alimentation en nutriments (Rashid et al., 2011) doit donc être intégrée dans l'analyse et prise en compte dans la planification des systèmes agricoles (Remans et al., 2014). Une alimentation non diversifiée conduit à la malnutrition à l'image du marasme et du kwashiorkor (Houndji et al., 2013 ; Lourme-Ruiz, 2016). La malnutrition compromet la bonne croissance physique et intellectuelle des jeunes enfants (Kayodé et al., 2012 ; Frempong et Annim, 2017).

Une enquête conduite au Burkina Faso, sous la tutelle du ministère de l'Agriculture, de l'Hydraulique et des Ressources Halieutiques (MAHRH) à travers la direction générale de l'Economie Rurale a révélé une prévalence de retard de croissance généralement élevée au niveau national $(>30 \%)$. Cette situation est plus sévère dans certaines régions notamment celle du Nord où la prévalence atteint 40,8\% (MAHRH, 2009). Les résultats rapportés par Billaz (2012) montrent que les familles rurales de la région du Nord, les adultes et les jeunes y compris, souffrent de déficits nutritionnels chroniques. Cette malnutrition est certainement liée à une mauvaise alimentation. Mais peu d'informations sont disponibles sur la consommation alimentaire des ménages pour mieux comprendre les causes de cette malnutrition. La connaissance d'information sur la consommation alimentaire dans la localité est nécessaire pour informer les acteurs travaillant dans le domaine de la sécurité alimentaire à mieux orienter leurs stratégies d'intervention.

C'est dans cette optique que la présente étude a été initiée pour évaluer la consommation alimentaire des ménages et identifier les déterminants essentiels qui permettent d'augmenter la diversité alimentaire des ménages et améliorer l'un des piliers de la sécurité alimentaire des populations locales.

\section{MATERIEL ET METHODES \\ Site d'étude}

L'étude s'est déroulée dans six (6) villages-sites du programme de recherche sur le changement climatique, l'agriculture et la sécurité alimentaire (CCAFS) que sont: Sillia, Ramdolla, Tibtenga, Basnéré, Lemnoogo et Tougou. Ces sites ont été sélectionnés par le programme CCAFS de façon participative avec la motivation que ces sites abritent déjà des partenaires de recherche connectés à un réseau de partenaires régionaux facilitant ainsi la mise à l'échelle des résultats de recherche (Perez et al., 2015). C'est aussi pour cette raison que notre choix s'est porté sur ces villages qui comptabilisent déjà des années d'expérience dans la participation aux 
activités de recherche pour assurer la qualité des données.

La Région du Nord est frontalière avec le Mali et comprend quatre provinces dont celle du Loroum, du Yatenga, de Zondoma et du Passoré. C'est une des régions les plus densément peuplées du pays, avec en moyenne 60 habitants/ $\mathrm{km}^{2}$ (Ouédraogo et Ripama, 2009) et qui est caractérisée par de fréquentes insécurités alimentaires (Billaz, 2012). La pluviométrie de cette région est très aléatoire et est marquée par l'imprévisibilité de chaque nouvelle campagne avec des pluies tardives ou des arrêts précoces de pluies avec des conséquences désastreuses sur les récoltes (Billaz, 2012).

Les projections sur les effectifs de la population de la région du Nord à partir des données du recensement général de la population effectué en 2006 ont donné une valeur de 1502527 habitants pour l'année 2015 (INSD, 2011). L'économie de la région repose essentiellement sur l'agriculture et l'élevage. Les populations sont engagées dans une agriculture de subsistance basée sur l'agriculture pluviale. Les cultures céréalières prédominantes sont le mil et le sorgho blanc, pratiquées dans $70 \%$ des cas sur sol dénudé (Marchal, 1986). Le sorgho rouge, le maïs et le riz sont des cultures marginales (Billaz, 2012). Les sols cultivables sont majoritairement représentés par les sols ferrugineux tropicaux et sont sujets à un ruissellement très important de l'ordre de 5 à $20 \%$ (Billaz, 2012). Introduit depuis les années 1960, le maraîchage représente une des principales activités de contre-saison dans la région (Ouédraogo, 1995) qui procure aux producteurs un revenu notable (Nebié, 1999). La région regorge d'énormes potentialités aurifères avec des terreaux d'exploitation artisanale de l'or un peu partout comme à Séguénéga, Guitti et Namissiguima (Capitant, 2017). Malgré cela, c'est une zone qui rencontre d'énormes difficultés pour nourrir sa population. Aucune des quatre provinces n'est à l'abri d'une année critique en termes de sécurité céréalière (Billaz, 2012) entraînant un fort taux de migration (34,9\%) vers les grands centres urbains (MAHRH, 2009).

\section{Enquête ménages}

Les données ont été collectées au moyen d'entretiens individuels auprès des chefs de ménages. Dans chaque village ciblé, un échantillon de 50 ménages a été constitué, donnant un effectif total de 300 ménages pour l'ensemble des six (6) villages-sites. L'unité d'observation a été le ménage représenté par le chef de ménage. Le ménage a été défini comme étant un groupe de personnes vivant ensemble comme une entité, qui conduisent ensemble les activités de production agricole et non agricole, qui participent ensemble aux dépenses du ménage et qui partagent les mêmes repas. Un questionnaire a été élaboré et axé sur la collecte d'informations démographiques (âge, sexe et niveau d'éducation du chef de ménage, taille du ménage), socio-économiques (les activités génératrices de revenus), sur la consommation alimentaire (différents groupes d'aliments consommés) et sur les systèmes de production (superficies emblavées, les cultures, la pratique de l'élevage). Les répondants ont été les chefs de ménages car dans ces localités comme partout ailleurs dans la région, ce sont eux qui prennent des décisions sur les questions majeures. Pour les questions en rapport avec la consommation alimentaire, les répondants ont été appuyés par les femmes responsables de la préparation du repas du ménage.

\section{Diversité alimentaire des ménages}

La diversité alimentaire se réfère aux aliments et groupes d'aliments consommés dans une période de référence donnée (Onyango, 2003 ; Rashid et al., 2011). Elle a été étudiée à travers le score de diversité alimentaire (SDAM) qui est une mesure de l'accès alimentaire des ménages sur une période de référence donnée (Ruel, 2002). La diversité alimentaire a été étudiée par plusieurs auteurs, mais avec des approches différentes quant à la période de référence 
et/ou au nombre de groupes d'aliments considérés (Bezerra et Sichieri, 2011 ; Rashid et al., 2011 ; Azadbakht et Esmaillzadeh, 2012 ; Workicho et al., 2016). L'approche utilisée au cours de la présente étude a été celle décrite par Hammond et al. (2017) qui est fondée sur les instructions de l'outil RHoMIS (Rural Household Multiple Indicator Survey) qui est un outil d'enquête pour la collecte rapide de données sur les ménages. L'outil RHoMIS est en partie une inspiration de l'approche décrite par Swindale et Bilinsky (2006), avec la particularité d'avoir une période de référence différente. Pour l'étude donc, une période de 4 semaines écoulées avant l'enquête a été retenue comme période de référence. Un des objectifs visés ici était de connaitre la fluctuation de la diversité alimentaire des ménages au cours de l'année. Ainsi, la première étape du travail a consisté à identifier dans les 12 mois de l'année, la période favorable, c'est-à-dire celle au cours de laquelle les ménages arrivent à satisfaire les besoins alimentaires de la famille et la période difficile correspondant à celle durant laquelle les ménages ne parviennent pas à satisfaire leurs besoins alimentaires.

Ensuite, il a été demandé aux répondants d'estimer leur consommation alimentaire en période favorable et en période difficile. Cette consommation a concerné les 12 groupes d'aliments recommandés par Swindale et Bilinsky (2006) pour lesquels les répondants devraient indiquer s'ils les consommaient quotidiennement, hebdomadairement, mensuellement ou s'ils n'en consommaient aucunement pas. La notation de Workicho et al. (2016) a été utilisée et les groupes d'aliment consommés au moins une fois par semaine ont reçu le score 1 et ceux qui n'ont pas été consommés ont reçu le score 0 . Le score de diversité alimentaire a été calculé en faisant la somme des scores des différents groupes d'aliments consommés (Ruel, 2002 ; Kennedy et al., 2011). La valeur du SDAM varie entre 0 et 12. Plus le SDAM est élevé plus la diversité alimentaire des ménages est élevée signifiant que plus de groupes d'aliments ont été consommés (Workicho et al., 2016).

Après la détermination du SDAM, les ménages ont été classés dans différents groupes selon le niveau du SDAM conformément à la classification utilisée par Kennedy et al. (2011) qui distingue trois groupes de ménages:

- le groupe de ceux qui consomment au plus trois (3) groupes d'aliments (SDAM faible);

- le groupe de ceux qui consomment entre 4 et 5 groupes d'aliments (SDAM moyen);

- et le groupe de ceux qui consomment au moins six (6) des groupes d'aliments (SDAM élevé).

\section{Analyses statistiques}

Les données collectées ont été analysées à l'aide du logiciel IBM SPSS Statistics 22. Des analyses de fréquence et de moyennes ont été effectuées pour décrire les caractéristiques des ménages, les scores de diversité alimentaire et les types de groupes d'aliments consommés. Les moyennes ont été calculées pour les variables quantitatives et la fréquence pour les variables catégorielles. Une analyse de régression logistique multinomiale a été effectuée pour prédire la probabilité pour qu'un ménage appartienne à un niveau de diversité alimentaire élevé ou faible. Le choix de ce modèle réside dans le fait qu'il soit bien adapté dans l'analyse des déterminants lorsqu'on a des variables explicatives qui contiennent des variables continues et des variables qualitatives (Folefack, 2015). La variable dépendante a été la diversité alimentaire avec ces trois sousgroupes (faible, moyen et élevé). Le niveau de diversité alimentaire moyen a été le niveau de référence. Le choix des variables explicatives a été basé sur une connaissance préalable des variables susceptibles d'influencer le niveau de diversité alimentaire. Les variables examinées comme déterminants de la diversité alimentaire ont été l'âge du chef de ménage ( $\leq$ 45 ans; entre 45-65 ans et > 65 ans selon la classification utilisée par Diiro et al. (2016) où les plus de 65 ans sont les inactifs); le sexe 
(masculin, féminin) ; le niveau d'éducation du chef de ménage (non scolarisé, niveau primaire, niveau post primaire) ; la taille du ménage $(<10$ membres ; $>10$ membres sur la base de la moyenne à partir des données d'enquête) ; les superficies emblavées $(<5,04$ ha et $>5,04$ ha sur la base de la moyenne à partir des données d'enquête) ; le nombre de plantes agricoles alimentaires produites $(\leq 6$ et $>6$ sur la base de la moyenne à partir des données d'enquête) ; le revenu tiré de l'élevage (oui, non) ; le revenu tiré de l'orpaillage (oui, non) ; le revenu tiré de la vente des produits agricoles (oui, non); le revenu tiré du maraîchage (oui, non) ; le revenu tiré du commerce (oui, non) et les transferts d'argent (oui, non).

\section{RESULTATS}

\section{Caractéristiques des ménages étudiés}

Les fréquences des répondants pour chaque catégorie des variables socioéconomiques et démographiques sont données dans le Tableau 1. Dans la zone d'étude, le type de ménage dominant est celui sous la responsabilité d'un homme. Sur les 300 ménages qui constituaient l'échantillon, 93,3\% étaient dirigés par des hommes et 6,7\% par des femmes. Les femmes chefs de ménage sont des veuves et des femmes dont les maris ont émigré. L'âge moyen des enquêtés est de $50,8(14,3)$ ans avec $16,7 \%$ étant des personnes avancées en âge au moins 65 ans d'âge. En plus, près de la moitié des répondants comptait au moins 10 membres dans leur ménage durant la période de l'enquête. La grande majorité (99\%) des répondants appartenait au groupe ethnique Mossi.

La population enquêtée est une population majoritairement illettrée car 83,3\% n'ont jamais fréquenté l'école formelle. Le niveau secondaire a été le niveau le plus élevé atteint au sein de la population enquêtée et concerne seulement $4,3 \%$ de celle-ci.

Les principales sources de revenu ont été la vente des produits des récoltes $(71,1 \%$ des répondants) et la vente des animaux de la ferme (52\% des répondants). D'autres sources de revenus sont également utilisées. Il s'agit du maraîchage $(48,7 \%$ des répondants), l'orpaillage $(17,1 \%$ des répondants), le commerce $(33,3 \%$ des répondants) et les transferts d'argent ( $33 \%$ des répondants). $\mathrm{La}$ majorité des répondants $(83,3 \%)$ possède des champs dont la superficie dépasse 2 ha avec $30,7 \%$ des répondants ayant des champs d'une superficie de 5 à 10 ha et $6 \%$ des répondants possèdent des champs de plus de 10 ha. Le nombre de plantes agricoles cultivées est pour la plupart des répondants situé entre 5 à 8 , avec $10 \%$ des répondants qui produisent plus de 9 cultures.

\section{Appréciation de la satisfaction des besoins alimentaires par les ménages enquêtés}

Il ressort de l'enquête que la majorité des ménages enquêtés $(85,3 \%)$ n'arrive pas à pourvoir aux besoins alimentaires de la famille au cours des douze mois de l'année. Les mois les plus difficiles varient en fonction $\mathrm{du}$ village (Figure 2). Cependant, dans l'ensemble, la période difficile ou période de soudure s'étend de juin à septembre et correspond à la période hivernale dans la région. A cette période, $54,7 \%$ des ménages estiment qu'il n'y a pas assez de nourriture pour toute la famille.

\section{Consommation alimentaire des ménages}

En période favorable, les ménages ont consommé en moyenne 7,3 $\pm 2,4$ aliments, avec un minimum de 1 et un maximum de 12 aliments $(n=300)$. Par contre, en période difficile, la valeur du SDAM est de 5,7 $\pm 2,4$.

Les aliments de base pour l'ensemble des ménages sont les céréales indépendamment de la saison (Figure 3). Les légumes, les féculents, la viande, le lait et les produits laitiers sont plus consommés pendant la période favorable comparativement à la période de soudure. Par contre, la consommation des fruits, des légumineuses, des huiles et du sucre reste stable au cours de l'année. Quel que soit le niveau de diversité alimentaire, les céréales et les légumineuses 
sont consommées par une proportion importante des ménages (Figures 4 et 5). Les ménages à diversité moyenne consomment en plus, pour la plupart, des légumes, du poisson, de l'huile et du sucre. Les ménages de diversité alimentaire élevée se distinguent par leur accès à d'autres produits supplémentaires tels les féculents, les fruits, la viande le lait et les produits laitiers.

Les aliments les plus fréquemment consommés ont été les céréales. Elles sont consommées tous les jours par $81 \%$ des ménages en période favorable et $82 \%$ de ces derniers en période difficile (Figures 6 et 7). Les légumes, le poisson et le sucre sont aussi fréquemment utilisés par les ménages. Bien que fréquente, la consommation de poisson se fait dans de très petites quantités essentiellement pour agrémenter les sauces. Le haricot (le niébé) est consommé plus de deux fois dans la semaine par presque la moitié des ménages $48,3 \%$ et $44,7 \%$ en période favorable et difficile respectivement. Les groupes d'aliments d'origine animale sont moins fréquemment consommés. La viande est consommée soit une fois dans la semaine $(32,7 \%)$ soit une fois dans le mois $(36,7 \%)$. Le lait et les produits laitiers sont tout aussi faiblement consommés. La majorité des répondants nous a confié qu'elle ne consomme presque jamais les œufs et ce, quelle que soit la saison de l'année.

\section{Déterminants de la diversité alimentaire des ménages}

L'analyse de régression logistique multinomiale a permis d'aboutir à des modèles concluants permettant de prédire la probabilité d'avoir un niveau de diversité alimentaire élevé ou faible. Les déterminants ont varié en fonction de la saison (Tableaux 2 et 3). En période de disponibilité alimentaire, les déterminants sont les transferts d'argent, l'orpaillage et le revenu tiré de la vente des produits d'élevage de bétail $(\mathrm{n}=293$; degré de liberté $=28$, chi-square $=121,89 ; \mathrm{p}<0,001$, nagelkerke $=0,46$ ). En période de soudure, les variables significatives au modèle sont : les transferts d'argent, l'orpaillage, le revenu tiré de la vente du bétail, la superficie totale cultivée, la vente des produits des récoltes et le nombre de cultures $(n=300$; degré de liberté $=28$, chi-square $=168,21 ; \mathrm{p}<0,001$, nagelkerke $=0,51)$. Les modèles expliquent $46 \%$ des variations de la diversité alimentaire des ménages en période favorable et $51 \%$ en période de soudure.

Le nombre de cultures alimentaires produites par les répondants n'a pas été associé à la diversité alimentaire des ménages durant la saison favorable. Par contre, il a été positivement lié à la faible diversité alimentaire des ménages pendant la période de soudure.

Un effet différencié des recettes agricoles a été observé entre les saisons de l'année. En effet, le revenu issu de la vente des produits agricoles n'a pas été lié à la diversité alimentaire pendant la période favorable mais a été positivement corrélée avec la faible diversité alimentaire pendant la période de soudure. Le revenu tiré de la vente du bétail, quant à lui, a été négativement corrélé avec le faible niveau de diversité alimentaire pendant la saison favorable et le lien n'a pas pu être clairement défini pour la période de soudure. Les résultats indiquent que les ménages qui tirent un revenu de la vente des produits agricoles ont eu une faible diversité alimentaire pendant la période de soudure et ceux qui n'ont pas de revenu tiré de l'élevage ont une probabilité élevée d'avoir une diversité alimentaire faible pendant la période défavorable.

Dans notre étude, seul le revenu issu de l'orpaillage a été positivement associé à la diversité élevée quelle que soit la saison. Ces résultats montrent que les ménages qui font de l'orpaillage ont une plus grande probabilité d'avoir une diversité alimentaire plus élevée. Cependant, les transferts d'argent ont été négativement corrélés avec la diversité élevée que ce soit pendant la période favorable ou la période de soudure. 
Tableau 1: Profil socio-économique des ménages ( $\mathrm{n}=300)$.

\begin{tabular}{|c|c|c|c|}
\hline Variables & Catégories & $\begin{array}{l}\text { Fréquences } \\
(\mathbf{n}=\mathbf{3 0 0})\end{array}$ & Pourcentage (\%) \\
\hline \multirow[t]{2}{*}{ Sexe des chefs de ménage } & Masculin & 280 & 93,3 \\
\hline & Féminin & 20 & 6,7 \\
\hline \multirow[t]{3}{*}{ Age des chefs de ménage (ans) } & $\leq 45$ & 114 & 38 \\
\hline & $45-65$ & 136 & 45,3 \\
\hline & $>65$ & 50 & 16,7 \\
\hline \multirow{4}{*}{$\begin{array}{l}\text { Statut matrimonial des chefs de } \\
\text { ménage }\end{array}$} & Marié $(e)=$ & 283 & $94,3 \%$ \\
\hline & célibataire $=$ & 2 & $0,7 \%$ \\
\hline & divorcé (e) & 1 & $0,3 \%$ \\
\hline & Veuf (e) & 14 & 4,7 \\
\hline \multirow[t]{4}{*}{$\begin{array}{l}\text { Niveau d'éducation des chefs de } \\
\text { ménage }\end{array}$} & $\begin{array}{l}\text { Jamais été à } \\
\text { l'école }\end{array}$ & 250 & 83,3 \\
\hline & Ecole primaire & 35 & 11,7 \\
\hline & $\begin{array}{l}\text { Ecole } \\
\text { secondaire }\end{array}$ & 13 & 4,3 \\
\hline & Supérieur & 2 & 0,7 \\
\hline \multirow[t]{5}{*}{ Taille du ménage } & $\leq 3$ & 12 & 4 \\
\hline & $4-5$ & 58 & 19,3 \\
\hline & $6-7$ & 47 & 15,7 \\
\hline & $8-9$ & 46 & 15,3 \\
\hline & $\geq 10$ & 137 & 45,7 \\
\hline \multirow[t]{6}{*}{ Superficie moyenne cultivée (ha) } & $\leq 2$ & 41 & 13,7 \\
\hline & $2-3$ & 54 & 18 \\
\hline & $3-4$ & 58 & 19,3 \\
\hline & $4-5$ & 37 & 12,3 \\
\hline & $5-10$ & 92 & 30,7 \\
\hline & $>10$ & 18 & 6 \\
\hline \multirow[t]{3}{*}{ Nombre de cultures } & $1-4$ & 37 & 12,3 \\
\hline & $5-8$ & 233 & 77,7 \\
\hline & $\geq 9$ & 30 & 10 \\
\hline \multirow[t]{2}{*}{ Pratique de l'élevage } & Oui & 287 & 95,7 \\
\hline & Non & 13 & 4,3 \\
\hline \multirow[t]{2}{*}{ Revenu issu de la vente du bétail } & Oui & 156 & 52 \\
\hline & Non & 144 & 48 \\
\hline \multirow{2}{*}{$\begin{array}{l}\text { Revenu issu de la vente des } \\
\text { cultures }\end{array}$} & Oui & 209 & 71,1 \\
\hline & Non & 85 & 28,9 \\
\hline \multirow[t]{2}{*}{ Maraîchage } & Oui & 146 & 48,7 \\
\hline & Non & 154 & 51,3 \\
\hline \multirow[t]{2}{*}{ Orpaillage } & Oui & 51 & 17,1 \\
\hline & Non & 248 & 82,9 \\
\hline \multirow[t]{2}{*}{ Commerce } & Oui & 100 & 33,3 \\
\hline & Non & 200 & 66,7 \\
\hline \multirow[t]{2}{*}{ Transferts d'argent } & Oui & 99 & 33 \\
\hline & Non & 201 & 67 \\
\hline
\end{tabular}




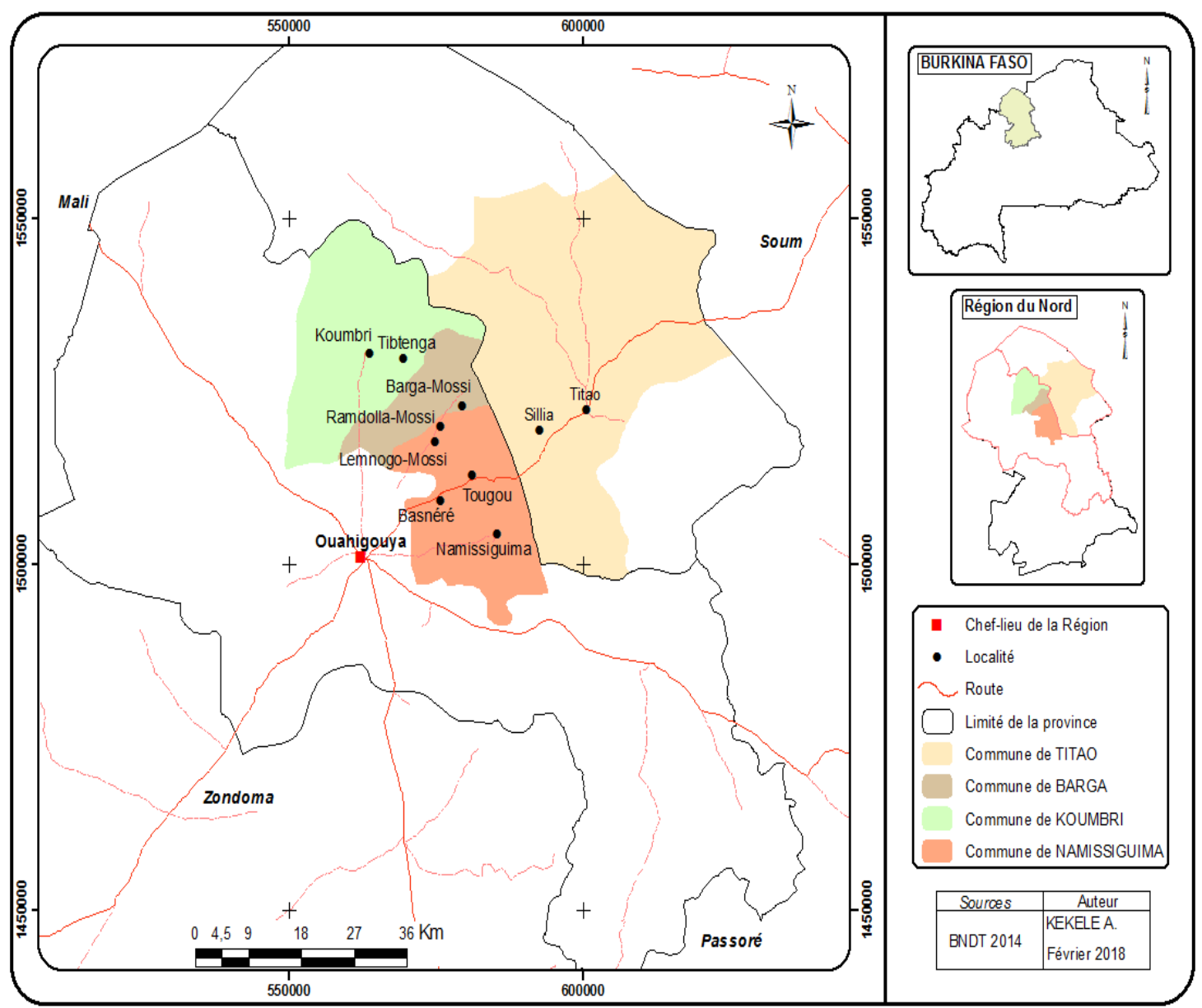

Figure 1: Carte de localisation des sites de l'étude.

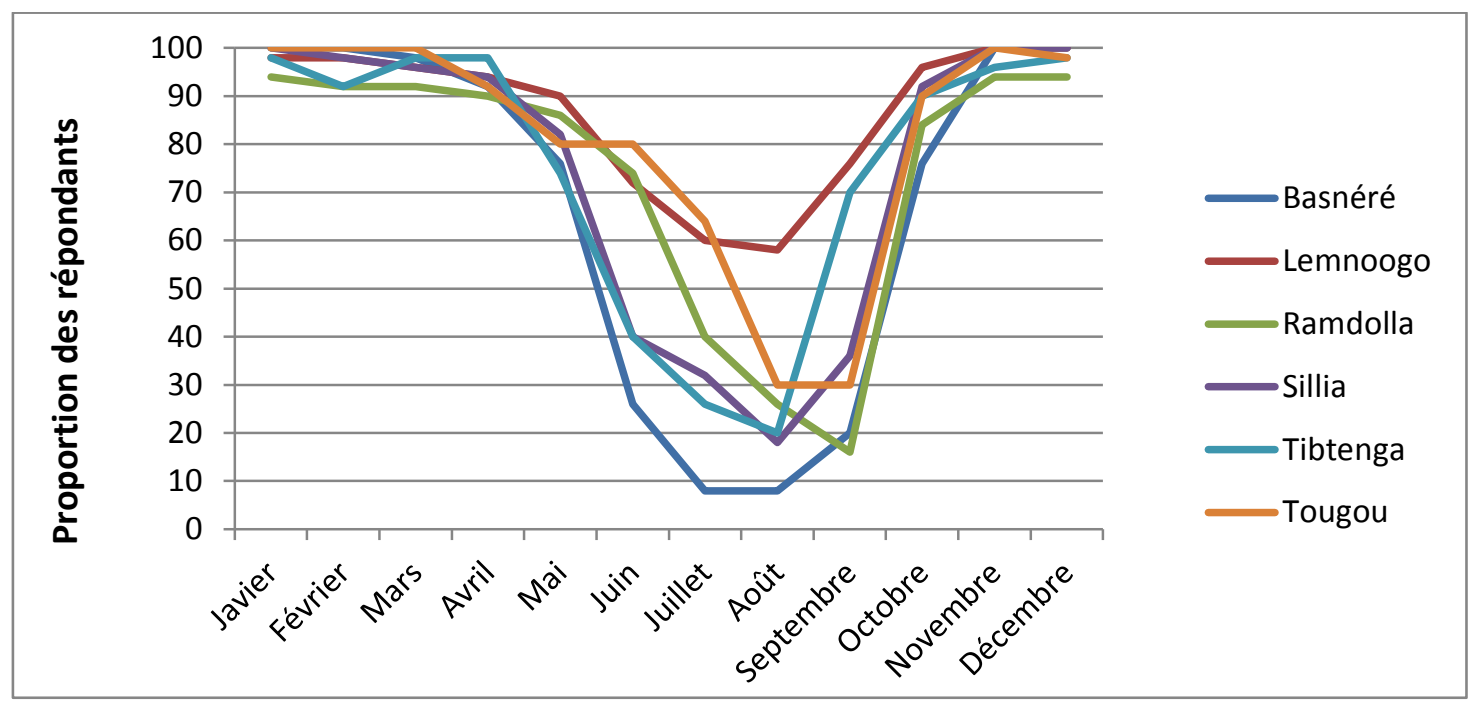

Figure 2: Proportion des ménages qui estiment que les besoins alimentaires de leur famille sont satisfaits. 


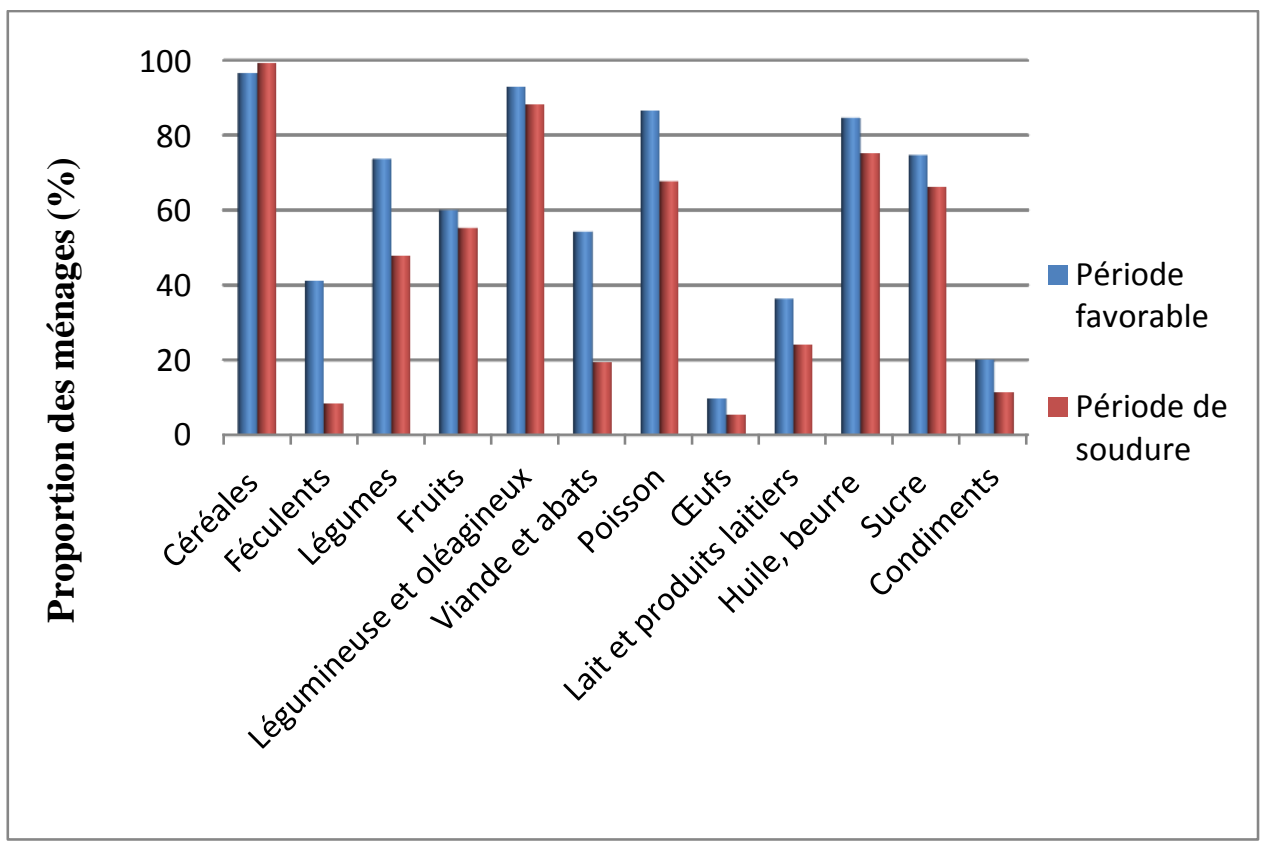

Figure 3: consommation des groupes d'aliments suivant les saisons de l'année.

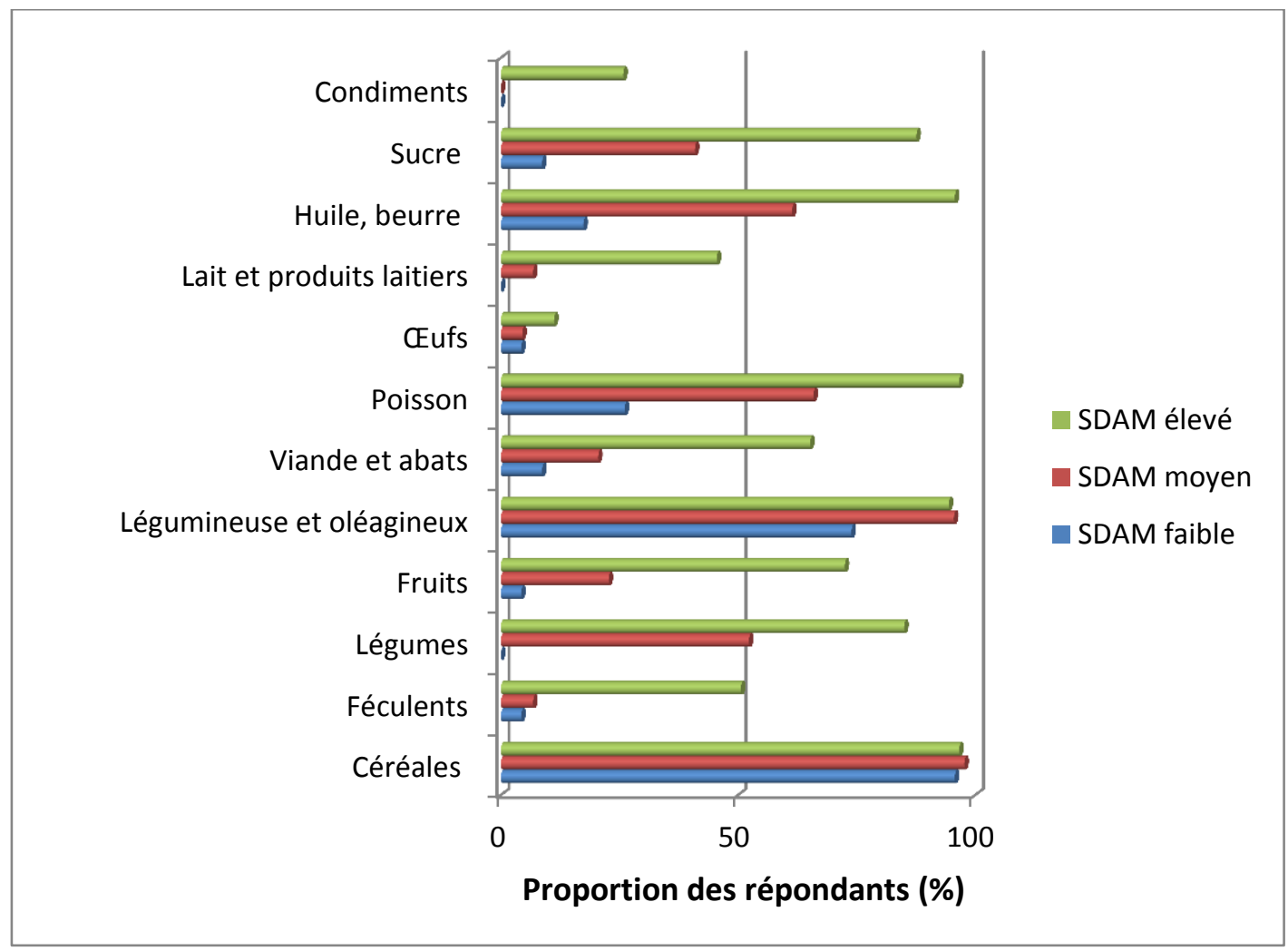

Figure 4 : Consommation des groupes d'aliments suivant le niveau de diversité alimentaire pendant la période favorable. 


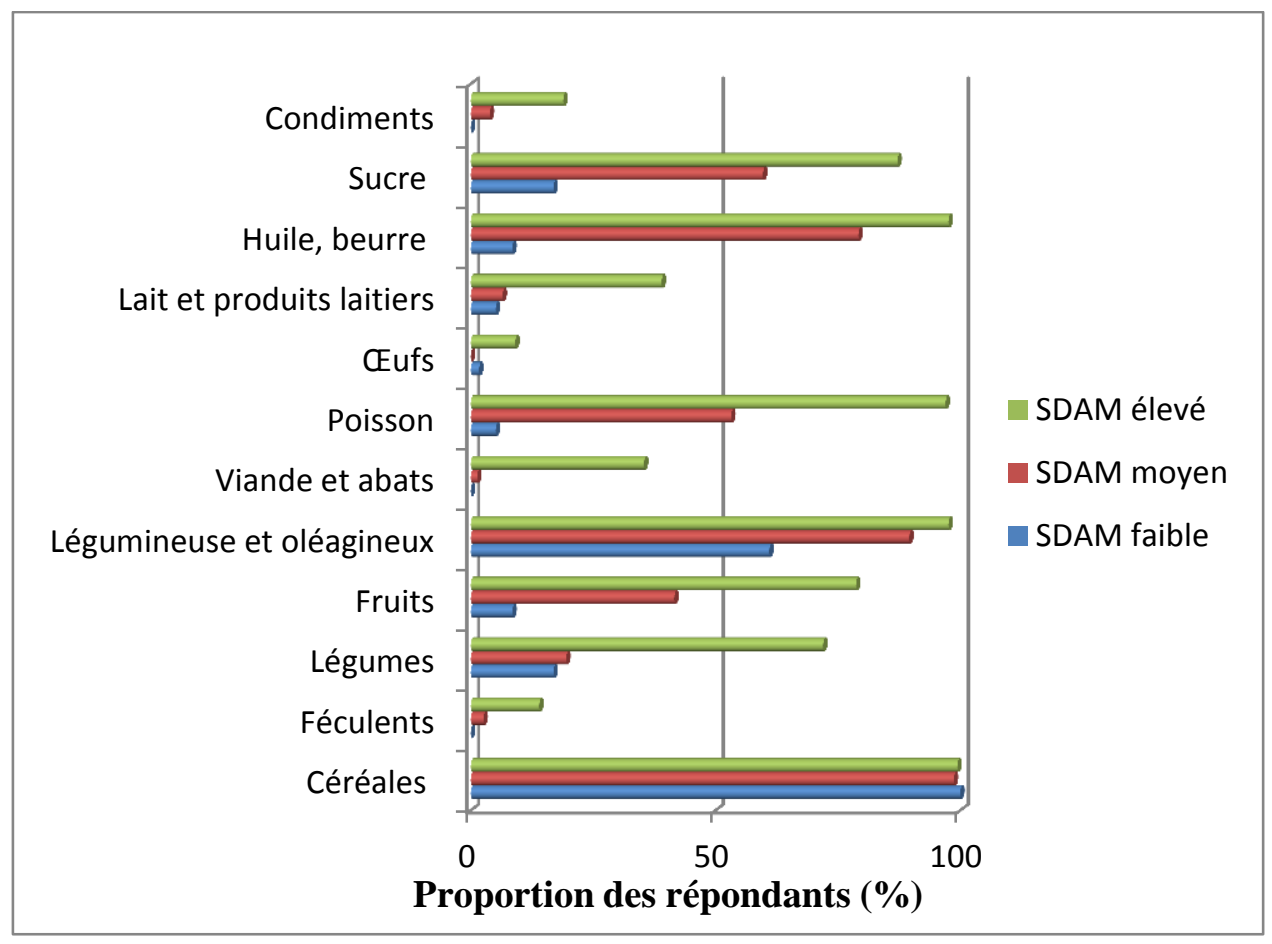

Figure 5: Consommation des groupes d'aliments suivant le niveau de diversité alimentaire pendant la période de la soudure.

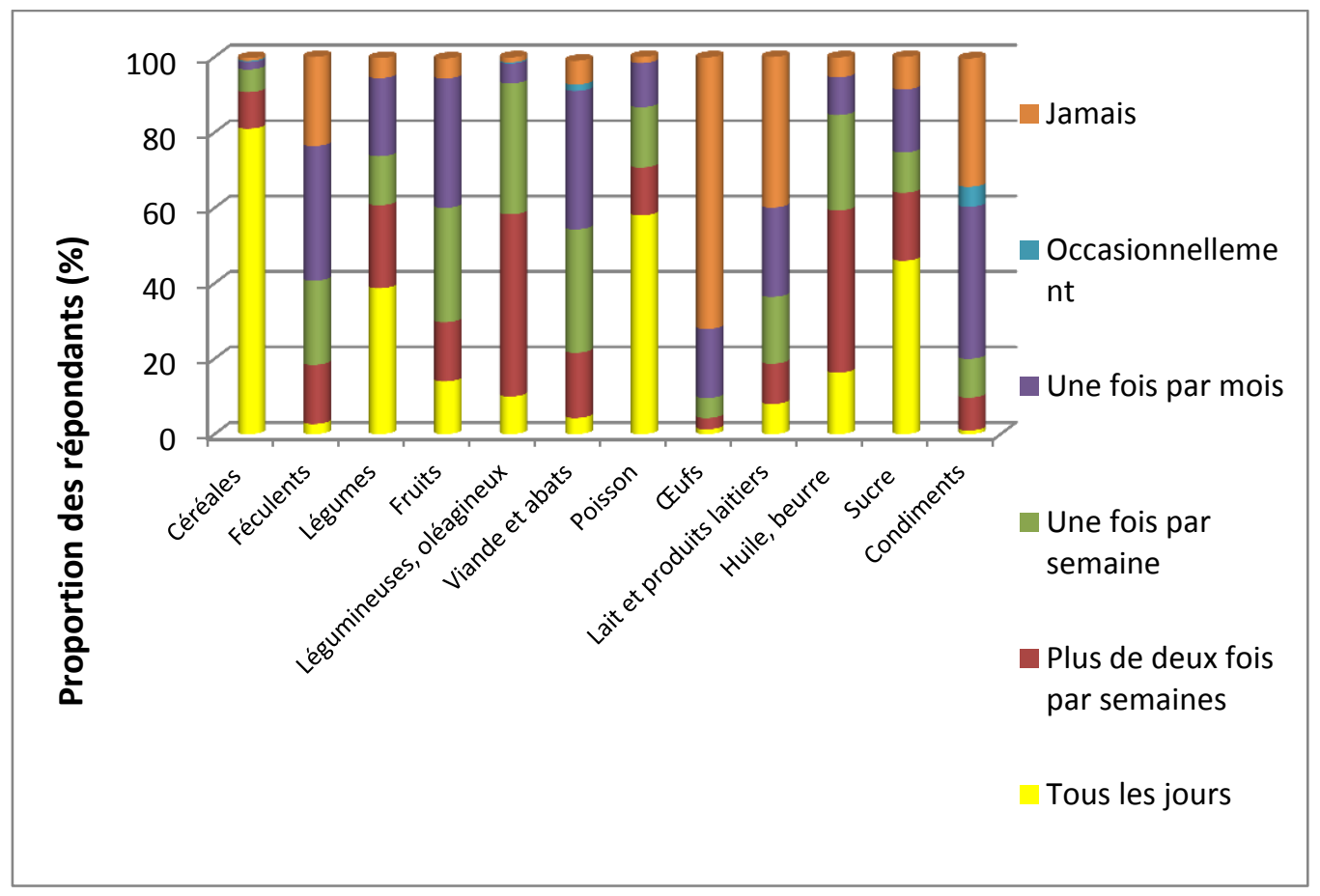

Figure 6: Consommation des groupes d'aliments en saison favorable (nombre des ménages $=300$ ). 


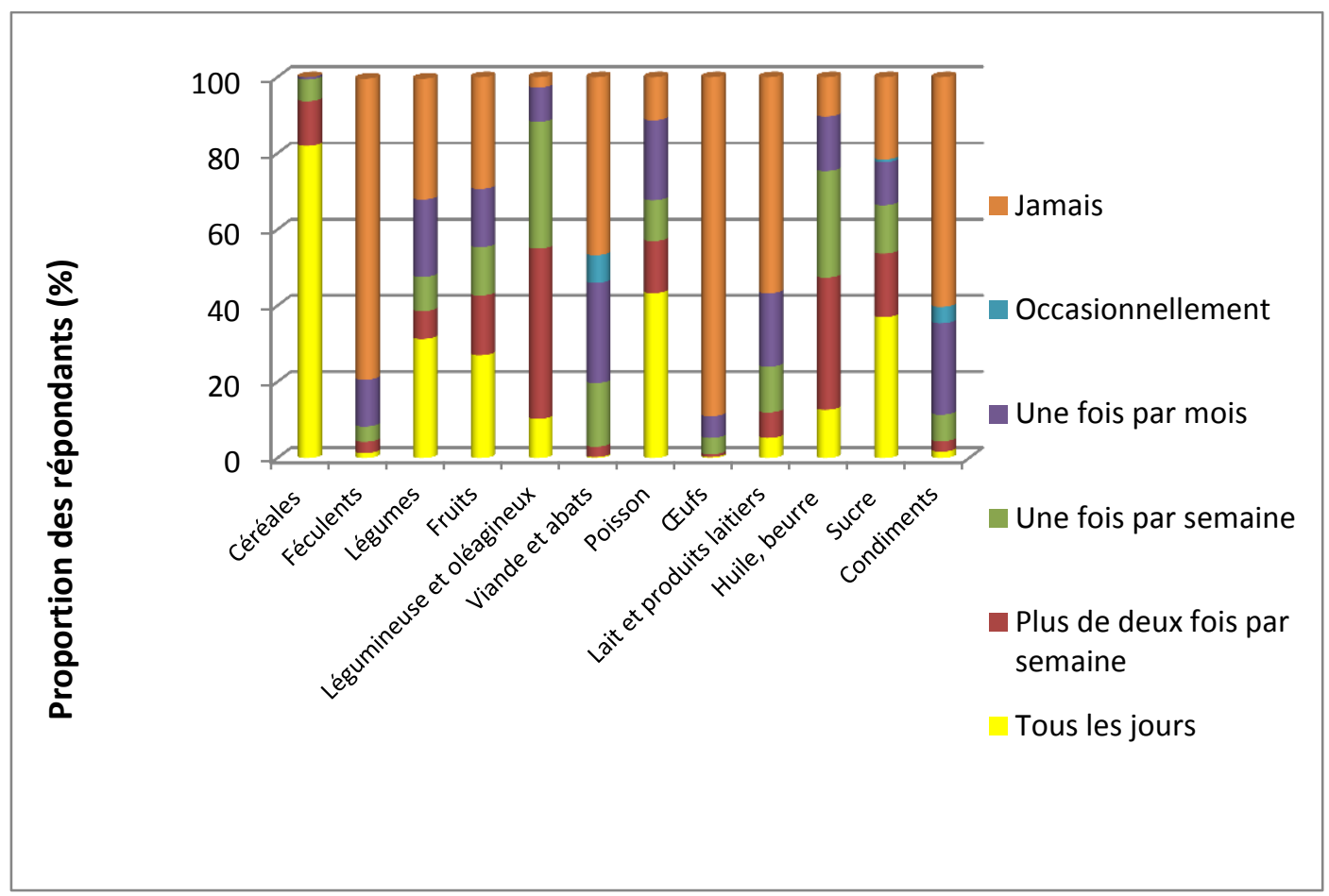

Figure 7: Consommation des groupes d'aliments en période de soudure (nombre des ménages $=300)$.

Tableau 2 : Déterminants de la diversité alimentaire des ménages pendant la période favorable.

\begin{tabular}{lcccccc}
\hline & \multicolumn{6}{c}{ Déterminants des ménages } \\
\hline & \multicolumn{2}{c}{ Faible diversité alimentaire } & \multicolumn{3}{c}{ Diversité alimentaire élevée } \\
\cline { 2 - 7 } Variables & $\mathbf{B}$ & $\mathbf{A O R}$ & $\mathbf{P}$ & $\mathbf{B}$ & AOR & $\mathbf{P}$ \\
\hline Sexe du chef de ménage & $-0,001$ & 0,99 & 0,56 & 0,008 & 1,01 & 0,99 \\
Age du chef de ménage & 0,689 & 1,99 & 0,47 & 1,33 & 3,77 & 0,02 \\
Niveau d'éducation du chef & $-0,10$ & 0,90 & 0,95 & $-1,69$ & 0,18 & 0,17 \\
de ménage & & & & & & \\
Taille du ménage & 1,11 & 3,03 & 0,17 & $-0,31$ & 0,73 & 0,47 \\
Superficie des champs & 0,952 & 2,59 & 0,23 & 0,416 & 1,516 & 0,35 \\
Nombre de cultures & $-0,22$ & 0,80 & 0,79 & $-0,87$ & 0,42 & 0,08 \\
Orpaillage & $-0,99$ & 0,37 & 0,26 & 2,20 & 9,01 & 0,001 \\
& & & & & & $* *$ \\
Maraîchage & 1,70 & 5,50 & 0,17 & 0,20 & 1,23 & 0,69 \\
Commerce & $-1,39$ & 0,25 & 0,09 & $-0,78$ & 0,46 & 0,13 \\
Vente produits agricole & 0,66 & 1,94 & 0,32 & $-0,24$ & 0,78 & 0,59 \\
Vente des produits & $-1,53$ & 0,22 & 0,02 & $-0,75$ & 0,47 & 0,07 \\
d'élevage & & & $*$ & & & \\
Transferts d'argent & $-0,58$ & 0,56 & 0,54 & $-1,60$ & 0,20 & 0,003 \\
& & & & & & $* *$ \\
\hline
\end{tabular}

B: coefficient ; AOR : odds ratio ajustés ; P : seuil de signification ; $* \mathrm{p}<0,05 ; * *: p<0,01$ 
Tableau 3 : Déterminants de la diversité alimentaire des ménages pendant la période de soudure.

\begin{tabular}{|c|c|c|c|c|c|c|}
\hline \multirow[b]{3}{*}{ Variables } & \multicolumn{6}{|c|}{ Déterminants des ménages } \\
\hline & \multicolumn{3}{|c|}{ Faible diversité alimentaire } & \multicolumn{3}{|c|}{ Diversité alimentaire élevée } \\
\hline & $\mathbf{B}$ & AOR & $\mathbf{P}$ & $\mathbf{B}$ & AOR & $\mathbf{P}$ \\
\hline Sexe du chef de ménage & $-1,13$ & 0,32 & 0,16 & 0,04 & 1,04 & 0,95 \\
\hline Age du chef de ménage & $-0,35$ & 0,70 & 0,63 & $-0,20$ & 0,82 & 0,72 \\
\hline $\begin{array}{l}\text { Niveau d'éducation du chef } \\
\text { de ménage }\end{array}$ & 1,96 & 7,10 & 0,15 & $-0,82$ & 0,44 & 0,39 \\
\hline Taille du ménage & $-0,56$ & 0,57 & 0,24 & $-0,42$ & 0,66 & 0,24 \\
\hline Superficie des champs & 0,89 & 2,43 & 0,08 & 1,08 & 2,94 & $0,004 * *$ \\
\hline Nombre de cultures & 1,21 & 3,34 & $\begin{array}{c}0,04 \\
*\end{array}$ & $-0,73$ & 0,48 & 0,06 \\
\hline Orpaillage & $-1,16$ & 0,31 & 0,05 & 1,96 & 7,06 & $0,003 * *$ \\
\hline Maraîchage & 0,60 & 1,82 & 0,31 & $-0,51$ & 0,60 & 0,20 \\
\hline Commerce & $-0,27$ & 0,77 & 0,61 & $-0,16$ & 0,86 & 0,68 \\
\hline Vente produits agricole & 1,15 & 3,17 & $\begin{array}{c}0,01 \\
*\end{array}$ & $-0,18$ & 0,83 & 0,65 \\
\hline Vente des produits d'élevage & $-1,60$ & 0,20 & $\begin{array}{l}0,00 \\
0 * *\end{array}$ & $-1,278$ & 0,279 & $0,001 * *$ \\
\hline Transferts d'argent & 1,02 & 2,76 & 0,10 & $-1,28$ & 0,28 & $0,001 * *$ \\
\hline
\end{tabular}

B: coefficient ; AOR : odds ratio ajustés ; P : seuil de signification ; $*$ p $<0,05 ; * *: p<0,01$

\section{DISCUSSION}

\section{Disponibilité et consommation alimentaire des ménages}

Il ressort de l'enquête que la disponibilité des aliments varie beaucoup durant l'année. Il y a une période favorable où la nourriture est suffisante et une période où il y a peu de nourriture disponible, connue sous l'appellation période de soudure. Une étude conduite à Tougou, l'un des sites de la présente investigation, a montré que les mois de juillet, août et septembre sont les mois de l'année au cours desquels de nombreux ménages souffrent de pénurie alimentaire (Somé et al., 2011). En effet, durant ces mois, l'essentiel de la nourriture consommée par le ménage a une provenance autre que leur propre exploitation, ce qui montre par ailleurs que ces mois sont des mois d'insécurité alimentaire pour les ménages (Somé et al., 2011).
Les résultats de la présente étude indiquent que les ménages enquêtés dépendent largement des céréales pour leur alimentation et que globalement, il y a une variabilité saisonnière dans la diversité alimentaire. La présence prépondérante des céréales dans les régimes alimentaires des ménages ruraux tels que ceux de notre étude a déjà été rapportée par d'autres auteurs (Bidisha et al., 2017). Contrairement aux céréales, les produits d'origine animale, les fruits et les légumes sont faiblement consommés. La faible consommation de ces aliments a été également relevée auprès d'autres communautés rurales au Soudan et en Ethiopie (Workicho et al., 2016 ; Khalid et al., 2017). Bien que la consommation des légumes soit en général faible, elle a été plus élevée pendant la période favorable. Cette situation s'expliquerait par deux raisons : 
- la disponibilité des légumes pendant la période favorable (saison sèche chaude et saison sèche froide) car la zone d'étude est une zone de production maraîchère, et que la période favorable coïncide avec la saison des cultures maraîchères ;

- la non prise en compte des légumesfeuilles sauvages par les répondants qui sont des ressources disponibles pendant la saison pluvieuse et qui constituent un aliment d'appoint de grande valeur nutritive pour les populations rurales.

Des efforts doivent être faits pour rehausser le niveau de connaissance des légumes-feuilles notamment les légumesfeuilles sauvages car ils pourraient contribuer à assurer l'équilibre alimentaire des populations pauvres grâce à leur composition protéique et minérale (Ocho-Anin Atchibri et al., 2012 ; Ngo Bogmis et al., 2018). Les régimes alimentaires fondés sur la consommation de céréales ou féculents comme aliments de base et pauvres en fruits et en produits d'origine animale sont habituels dans les pays à faible revenu (Jones et al., 2014). Le risque d'une telle alimentation est la déficience en micronutriments et par conséquent, la malnutrition (Bidisha et al., 2017) et un mauvais état de santé (Payne et al., 2012 ; Mc Martin et al., 2013).

\section{Déterminant de la diversité alimentaire du ménage}

L'âge, le sexe et le niveau d'éducation du chef de ménage ainsi que la taille du ménage n'ont pas été des déterminants significatifs de la diversité alimentaire dans cette étude contrairement aux résultats rapportés par d'autres études (Jones et al., 2014 ; Workicho et al., 2016).

Nos résultats montrent que le nombre d'espèces alimentaires produites n'est pas positivement associé à une diversité alimentaire élevée et ce, quelle que soit la période de l'année. Ce résultat est en contradiction avec celui de Jones et al. (2014) qui ont trouvé une association positive entre la diversification des cultures et la diversité alimentaire. Mais similaire à celui observé par (Lourme-Ruiz et al., 2016) dans une autre région du Burkina Faso (Hauts Bassins). L'explication donnée par ces auteurs serait que :

- Les systèmes de production sont peu diversifiés en se référant aux groupes d'aliments car deux ou trois cultures peuvent appartenir au même groupe d'aliment ;

- Une partie importante de la production non céréalière est vendue et non consommée.

L'existence d'une relation positive entre la situation économique des ménages et la diversité alimentaire élevée a été démontrée au Brésil par Bezerra et Sichieri (2011). Des études spécifiques ont montré que la diversité alimentaire était positivement liée au revenu tiré de l'élevage (Jodlowski et al., 2016; Longin et Wurschum, 2016), de la vente des produits agricoles (Jones et al., 2014) et aux transferts d'argent (Bhalla et al., 2018). Cependant, nos résultats montrent que les revenus tirés de la vente des produits agricoles et des animaux ainsi que les transferts d'argent, ne sont pas positivement corrélés avec la diversité alimentaire élevée. Mais que le revenu tiré de la vente des animaux est négativement associé avec la diversité alimentaire. Ce résultat n'est pas aussi surprenant quand on sait que, pour qu'un revenu puisse impacter la diversité alimentaire, il faut que non seulement le cash obtenu atteigne un certain seuil mais aussi que cet argent soit dédié à l'achat des aliments tels que les légumes, les fruits, les légumineuses, les fruits et les produits d'origine animale pour diversifier les régimes alimentaires (Jones et al., 2014 ; Lourme-Ruiz et al., 2016). Selon ce dicton mossi (MAHRH, 2009) qui dit «Si tu ne manges pas un jour, personne ne le saura mais si tu passes une nuit à la belle étoile parce que tu n'as plus de maison tout le monde en sera informé», les revenus obtenus seraient probablement affectés à des dépenses autres (santé, dépenses liées à la scolarité, etc.) que les dépenses de l'alimentation. Dans notre étude, seul le revenu de l'orpaillage a été positivement associé à la diversité alimentaire élevée indépendamment de la saison. Ces constats suggèrent deux hypothèses : 
1. Dans les pays en voie de développement où le souci majeur demeure le déficit alimentaire (Torheim et al., 2004), les ménages accordent une très grande importance aux quantités d'aliment disponibles mais sont beaucoup moins préoccupés par rapport à la diversité de l'alimentation. Ils ont tendance à privilégier la consommation des aliments à haute densité énergique inversement associée à la diversité alimentaire et à la consommation des fruits et légumes (Azadbakht et Esmaillzadeh, 2012). L'équilibre nutritionnel n'est donc pas une préoccupation majeure, et peut même être considéré comme un luxe.

2. Les revenus des ménages pourraient être classés en plusieurs types :

a. Des revenus résultant d'une vente de produits de la ferme (céréales, bétail, volailles, etc.) suite à des dépenses planifiées ou à des besoins urgents ou importants de liquidité (achat de vivres en période de crise, frais de santé, frais liés à des cérémonies coutumières, dépenses liées à la scolarité des enfants, besoins de déplacement vers des destinations lointaines, etc.) ; ces revenus sont liés à des décisions prises par le chef de ménage. Certains auteurs abondent dans le même sens en montrant, que c'est essentiellement en cas de besoins urgents de liquidité monétaire, que les ménages procèdent à une décapitalisation de leur capital physique se traduisant soit par la vente des animaux soit par la vente des céréales (Gondard-Delcroix et Rousseau, 2004; Janin, 2004).

b. Des transferts d'argent représentant un soutien plus ou moins régulier d'un membre de la famille en migration dans une autre localité ; le caractère relativement régulier de ce type de revenu laisse penser que son utilisation peut être planifiée à l'avance (Barrett et al., 2001).

c. Des revenus à caractère plus aléatoire, en l'occurrence ceux résultant d'activités minières ; ces revenus ne sont pas liés à un événement ou à un besoin urgent et sont de nature irrégulière ; leur utilisation est donc très libre et cela peut justifier qu'ils interviennent dans l'amélioration de la qualité de l'alimentation (Barrett et al., 2001).
Des revenus n'entrant dans aucune des catégories ci-dessus, comme ceux issus d'activités génératrices de revenus (cultures maraîchères, commerce, production et la vente de beurre de karité...) ; on peut suggérer que l'utilisation de tels revenus est similaire à celle de la catégorie (b).

Malgré le fait que les modèles établis soit significatifs, la part de variabilité expliquée par les déterminants, respectivement $46 \%$ et $51 \%$ témoigne de l'existence d'autres facteurs qui influencent la diversité alimentaire dans la localité. En effet, des études conduites en Ethiopie et au Soudan ont montré que certains groupes d'aliments n'étaient pas consommés à cause de certaines considérations culturelles et de la méconnaissance de l'importance d'une alimentation variée (Khalid et al., 2017). Des ménages interrogés sur leur faible consommation d'œufs ont mentionné à 85,3\% des raisons culturelles et seulement $14,7 \%$ ont mentionné la faiblesse des moyens financiers ou des allergies. Dans leur communauté, la consommation des œufs est autorisée pour les hommes et interdite pour les femmes et les enfants (Khalid et al., 2017).

\section{Conclusion}

Le régime alimentaire journalier est faiblement diversifié dans la plupart des ménages selon les résultats de cette étude. Les aliments de base pour l'ensemble des ménages sont généralement les céréales qui servent à faire le «tô », pâte épaisse préparée à base de la farine de céréale et de l'eau, accompagné d'une sauce préparée à base de légumes et agrémentée avec du poisson sec réduit en poudre. Les ménages à diversité alimentaire moyenne consomment en plus, les légumineuses et les huiles. Les ménages de diversité alimentaire élevée se distinguent par la consommation des féculents, de la viande, des fruits, le lait et les produits laitiers. La pauvreté des régimes alimentaires en produits d'origine animale, en fruits et en légumes, engendrerait une déficience alimentaire en micronutriments. Cette déficience nutritionnelle peut avoir des incidences négatives sur la santé des enfants, voire des 
personnes adultes. Cette situation interpelle à des prises de mesure pour améliorer la situation alimentaire des ménages. Nos résultats montrent que les revenus à part celui tiré de l'orpaillage n'ont pas été positivement corrélés avec une diversité alimentaire élevée. Cela suggère donc la prise de mesures pour sensibiliser et encourager la consommation d'aliments diversifiés en mettant l'accent sur les problèmes de santé que cela causerait. Les résultats peuvent également aider les différents programmes de sécurité alimentaire et nutritionnelle intervenant dans la zone à mieux orienter leurs actions et sensibiliser sur le bienfait d'une alimentation diversifiée.

\section{CONFLIT D'INTERETS}

Les auteurs déclarent qu'il n'y a aucun conflit d'intérêts concernant le manuscrit.

\section{CONTRIBUTIONS DES AUTEURS}

SS a analysé et interprété les données puis préparé le manuscrit. AA a mené le design des instruments d'enquête pour l'étude, a supervisé la collecte de données et fait la revue du manuscrit. AJN a fait une révision critique du manuscrit. Tous les co-auteurs ont participé à la relecture du manuscrit et approuvé la version finale.

\section{REMERCIEMENTS}

Nous sommes reconnaissants aux répondants des sites de l'étude dans la région du Nord au Burkina Faso pour leur participation dans cette étude. En outre, nous remercions Viviane Yameogo pour la coordination de la collecte et la saisie de données. Les auteurs sont seuls responsables des opinions exprimées dans ce manuscrit.

\section{REFERENCES}

Azadbakht L, Esmaillzadeh A. 2012. Dietary energy density is favorably associated with dietary diversity score among female university students in Isfahan. Nutrition,

28: 991-995. http://dx.doi.org/10.1016/j.nut.2011.12.0 17
Barrett CB, Reardon T, Webb P. 2001. Nonfarm income diversification and household livelihood strategies in rural Africa: Concepts, dynamics, and policy implications. Food Policy, 26: 315-331.

Bezerra I, Sichieri R. 2011. Household food diversity and nutritional status among adults in Brasil. International Journal of Behavioral Nutrition and Physical Activity, 8: 22. DOI:10.1186/1479-58688-22

Bhalla G, Handa S, Angeles G, Seidenfeld D. 2018. The effect of cash transfers and household vulnerability on food security in Zimbabwe. Food Policy, 74: 82-99. https://doi.org/10.1016/j.foodpol.2017.1 1.007

Bidisha SH, Khan A, Imranc K, KhondkerB.H, Suhrawardy G.M. 2017. Role of credit in food security and dietary diversity in Bangladesh. Economic Analysis and Policy, 53: 3345.

http://dx.doi.org/10.1016/j.eap.2016.10.0 04

Billaz R. 2012. La lute contre les aléas climatiques au Burkina Faso. Acquis et défis de l'agro-écologie : le cas de la région du Nord. In La Grande Muraille Verte : Capitalisation des Recherches et Valorisation des Savoirs Locaux (en ligne). IRD Editions: Marseille ; 263315. (Généré le 18 janvier 2018). Disponible sur internet < http:/books.openedition.org/irdeditions/3 301>.

Capitant S. 2017. Les populations à l'assaut des mines: économie rurale, de la contestation minière au Burkina Faso. In Anthropologie des Prédations Foncières. Entreprises Minières et Pouvoirs Locaux. Leclerc-Olive M (ed). Edition des Archives Contemporaines: Paris (France). www.archivescontemporaines.com Diiro G, Petri M, Zemadim B, Sinare B, Dicko M, Traore D, Tabo R. 2016. 
Gendered Analysis of Stakeholder Perceptions of Climate Change, and the Barriers to its Adaptation in Mopti, Region in Mali. Research Report no. 68. Patancheru 502 324. Telangana, India: International. Crops Research Institute for the Semi-Arid Tropics, $52 \mathrm{p}$.

Frempong RB, Annim SK. 2017. Dietary diversity and child malnutrition in Ghana. Heliyon, 3. http://dx.doi.org/10.1016/j.heliyon.2017. e00298

Gondard-Delcroix C, Rousseau S. 2004. Vulnérabilité et Stratégies durables de gestion des risques : Une étude appliquée aux ménages ruraux de Madagascar. Développement Durable et Territoires. DOI:10.4000/developpementdurable.114 3

Hammond J, Fraval S, Etten J.V, Suchini J.G, Mercado L, Pagella T, Frelat R, Lannerstad M, Douxchamps S, Teufel N, Valbuena D, vanWijk MT. 2017. The Rural Household Multi-Indicator Survey (RHoMIS) for rapid characterisation of households to inform climate smart agriculture interventions: Description and applications in East Africa and Central America. Agricultural Systems, 151: 225-233. DOI: http://dx.doi.org/10.1016/j.agsy.2016.05. 003

Houndji BVS, Bodjrenou SF, Londji SBM, Ouetchehou R, Acakpo A, Amouzou KSSE, Hounmenou D. 2013. Amélioration de l'état nutritionnel des enfants âgés de 6 à 30 mois à Lissèzoun (Centre-Bénin) par la poudre de feuilles de Moringa oleifera (Lam.). Int. J. Biol. Chem. Sci., 7(1): 225-235. DOI : http://dx.doi.org/10.4314/ijbcs.v7i1.19

INSD (Institut national de la statistique et de la démographie). 2011. Recensement Général de la population et de l'Habitation de 2006. Fichiers des villages du Burkina Faso. INSD, septembre 2011, 403p.

Janin P. 2004. Gestion spatio-temporelle de la soudure alimentaire dans le Sahel burkinabé. In: Tiers-Monde, tome 45, $\mathrm{n}^{\circ} 180,2004$. ONG : les pièges de la professionnalisation. Tiers-Monde, 45: 909-933. DOI : 10.3406/tiers.2004.5539.

Folefack AJJ. 2015. The determinants for the adoption of compost from household waste for crop production by farmers living nearby Yaoundé, Cameroon: descriptive and logit model approaches of analysis. Int. J. Biol. Chem. Sci. 9(1): 308-328.

DOI: http://dx.doi.org/10.4314/ijbcs.v9i1.28

Jodlowski M, Winter-Nelson A, Baylis K, Goldsmith PD. 2016. Milk in the Data: Food Security Impacts from a Livestock Field Experiment in Zambia. World Development, 77: 99-114. http://dx.doi.org/10.1016/j.worlddev.201 5.08 .009

Jones AD, Shrinivas A, Bezner-Kerr R. 2014. Farm production diversity is associated with greater household dietary diversity in Malawi: Findings from nationally representative data. Food Policy, 46: 112.

DOI: http://dx.doi.org/10.1016/j.foodpol.2014. 02.001

Kayodé APP, Akogou FUG, Amoussa Hounkpatin W, Hounhouigan DJ. 2012. Effets des procédés de transformation sur la valeur nutritionnelle des formulations de bouillies de complément à base de sorgho. Int. J. Biol. Chem. Sci., 6(5): 2192-2201.

DOI: http://dx.doi.org/10.4314/ijbcs.v6i5.25

Khalid FA, Ali AKM, Ali SA, Mosmar ZYA, Salih SSM, Salman TK, Desogi MA, Soghaier MA, Mohammed EE, Mohammed AA. 2017. Households' dietary habits and food consumption patterns in Hamishkoreib locality, Kassala State, Sudan. J Ethn Foods, 4: 
181-186.

DOI:

http://dx.doi.org/10.1016/j.jef.2017.08.0

09

Kennedy G, Ballard T, Dop MC. 2011. Guidelines for measuring household and individual dietary diversity. Rome, Italy: FAO. ISBN 978-92-5-106749-9

Longin CFH, Wurschum T. 2016. Back to the future-tapping into ancient grains for food diversity. Trends in Plant Science, 21:

731-737. http://dx.doi.org/10.1016/j.tplants.2016.0 5.005

Lourme-Ruiz A, Dury S, Martin-Prével Y. 2016. Consomme-t-on ce que l'on sème ? Relations entre diversité de la production, revenu agricole et diversité alimentaire au Burkina Faso. Cahiers Agricultures, 25: 11p. DOI : https://doi.org/10.1051/cagri/2016038

Marchal JY. 1986. Vingt ans de lutte antiérosive au nord du Burkina Faso. Cah. ORSTOM, sér. Pédol., XXII: 173180.

McMartin SE, Jacka FN, Colman I. 2013. The association between fruit and vegetable consumption and mental health disorders: Evidence from five waves of a national survey of Canadians. Preventive Medicine, 56: 225-230. DOI : http://dx.doi.org/10.1016/j.ypmed.2012.1 2.016

MAHRH (Ministère de l'Agriculture, de l'Hydraulique Et des Ressources Halieutiques). 2009. Enquête Nationale sur l'Insécurité Alimentaire et la Malnutrition Rapport Définitif, 193 p.

Nebié O. 1999. Approvisionnement urbain et développement des cultures maraichères dans la région deOuagadougou (Burkina Faso). Les cahiers d'Outre-Mer, 52: 257-274. DOI : https://doi.org/10.3406/caoum.1999.373 3

Ngo Bogmis MN, Ngwa FA, Manga GA. 2018. Evaluation nutritionnelle de la morelle africaine au Cameroun. Int. J. Biol. Chem. Sci., 12(1): 62-74. DOI : https://dx.doi.org/10.4314/ijbcs.v12i1.5

Ocho-Anin Atchibri AL, Soro LC, Kouame C, Agbo EA et Kouadio KKA. 2012. Valeur nutritionnelle des légumes feuilles consommés en Côte d'Ivoire. Int. J. Biol. Chem. Sci., 6(1): 128-135. DOI: http://dx.doi.org/10.4314/ijbcs.v6i1.12

Onyango AW. 2003. Dietary diversity, child nutrition and health in contemporary African Communities. Comparative Biochemistry and Physiology Part A 136: 61-69. DOI: 10.1016/S10956433(03)00071-0

Ouédraogo M. 1995. Histoire et conséquences de l'introduction du maraichage en zone soudano-sahélienne au Burkina Faso. In Innovation et sociétés : quelles agricultures? Quelles innovations? : 2. Les diversités de l'innovation, Chauveau J-P, Yung JM (eds). CIRAD : Montpellier (France); 257-263. Séminaire International d'Economie Rurale, Montpellier (France), 1316/09/1993.

Ouédraogo M, Ripama T. 2009. Recensement général de la population et de l'habitation (RGPH) de 2006. Analyse des résultats définitifs. Thème 2 : état et structure de la population. Institut national de la statistique et de la démographie, $\quad 180 \quad \mathrm{p}$ http://www.cns.bf/IMG/pdf/th_2_etat_et _structure_de_la_population_f.pdf

Payne ME, Steck SE, George RR, David C, Steffens DC. 2012. Fruit, Vegetable, and Antioxidant Intakes Are Lower in Older Adults with Depression. J Acad Nutr Diet., 112: 2022-2027. doi: 10.1016/j.jand.2012.08.026

Perez C, Jones EM, Kristjanson P, Cramer L, Thornton PK, Förch W, Barahona C. 2015. How resilient are farming households and communities to a changing climate in Africa? A gender- 
based perspective. Global Environmental

Change, 34: 95-107. DOI: http://dx.doi.org/10.1016/j.gloenvcha.20 15.06.003

Rashid DA, Lisa C, Smith LC, Rahman T. 2011. Determinants of Dietary Quality: Evidence from Bangladesh. World Development, 39: 2221-2231. DOI: 10.1016/j.worlddev.2011.05.022

Remans R, Wood SA, Saha Nd, Anderman TL, DeFries RS. 2014. Measuring nutritional diversity of national food supplies. Global Food Security, 3: 174182. DOI: http://dx.doi.org/10.1016/j.gfs.2014.07.0 01

Ruel MT. 2002. Is dietary diversity an indicator of food security or dietary quality? A review of measurement issues and research needs. FCND (International Food Policy Research Institute) discussion paper no. 140. International Food Policy Research Institute (IFPRI), Washington D.C.

Somé L, Sissoko K, Zougmoré R, Traoré B, Amadou M, Moussa AS, Förch W, Garlick C, Ochieng S, Kristjanson P, Thornton PK. 2011. Résumé des résultats des enquêtes de base niveau ménage - site de Tougou, Burkina Faso. Programme de recherche du CGIAR sur le Changement Climatique, l'Agriculture et la Sécurité Alimentaire (CCAFS). Copenhague, Danemark. Disponible en ligne sur www.ccafs.cgiar.org

Swindale A, Bilinsky P. 2006. Household Dietary Diversity Score (HDDS) for Measurement of Household Food Access: Indicator Guide (v.2). Washington, D.C.: FHI 360/FANTA.

Torheim LE, Ouattara F, Diarra MM, Thiam FD, Barikmo I, Hatløy A, Oshaug A. 2004. Nutrient adequacy and dietary diversity in rural Mali: association and determinants. European Journal of Clinical Nutrition, 58: 594-604. DOI: 10.1038/sj.ejcn.1601853

Workicho A, Belachew T, Feyissa GT, Wondafrash B, Lachat C, Verstraeten R, Kolsteren P. 2016. Household dietary diversity and Anima Source Food consumption in Ethiopia: evidence from the 2011 Welfare Monitoring Survey. Public Health, 16. DOI : 10.1186/s12889-016-3861-8. 